\title{
SPN-Based Performance analysis of troupes' behaviors of sharing theatres
}

\author{
Zhiguo Hong1, Ying Li1, Wei Jiang123, Yongbin Wang123 \& Shuang Feng1 \\ 1Faculty of Science and Engineering, Communication University of China, Beijing 100024, China \\ 2Key Laboratory of Acoustic Visual Technology and Intelligent Control System, Ministry of \\ Culture, Beijing 100024, China \\ 3Beijing Key Laboratory of Modern Entertainment Technology, Beijing 100024, China \\ e-mail: hongzhiguo@cuc.edu.cn
}

Keywords: petri nets, performance analysis, troupe, theatre.

\begin{abstract}
At present, manage systems for entertainment performance chains are built to work as agents to collaborate all participants, such as troupes, theatres and audience etc. Analyzing and mining the mechanism of behaviors of participants is an important issue which can be important gudie to establish such manage systems. On the basis of analyzing troupes' interactive process with audience and theatre, this paper constructed a Stochastic Petri Net (SPN) model for describing the behaviors of two troupes. Furthermore, average time delay was chosen as the performance index to evaluate the performance of the model with Stochastic Petri Net Package (SPNP) 6.0. For different parameters of number of presentations, size of theatre space, space requirements, various trends and numerical results are derived thereby. The methodology of modeling and simulation in this paper can be further used to study the performance of manage systems for entertainment performance chains.
\end{abstract}

\section{Introduction}

With the rapid development of theatre industry recently, manage systems for entertainment performance chains are built to work as agents to collaborate all participants, such as troupes, theatres and audience etc. For such manage systems, the average time delay of the whole process of presentations acted by troupes plays an important role. Average time delay is the key index of manage system which would influence the experiences of both troupes and audience. Further, three phenomena of participants are collaboration, parallelism and competition in using shared theatre resource.

Nowadays, methodologies of performance evaluation for collaborative system mainly take three ways, which are direct measurement, mathematic modeling [1, 2, 3, 4], and simulation. Mathematic modeling and simulation are undoubted useful methodologies. Consequently, how to model and evaluate the behaviors of participants is an important issue which offers theoretical foundations for building and deploying manage systems for entertainment performance chains. Petri nets, which was first developed in 1962 by C.A. Petri in his PhD. dissertation, is powerful in modeling concurrent, distributed, asynchronous behaviors of a system [5]. With algebra theory and the net theory as its mathematical basis, the Petri nets theory has been successfully employed to describe various relations and behaviors of the discrete event system and communication networks [6, 7].

The paper is organized as follows. In the next section, we investigate the timing diagram by considering the interactive process among troupes, theatre and audiences. Section 3 constructs the Stochastic Petri Net (SPN) [8] model of two troupes' behaviors of sharing theatre with different resource needs. In Section 4, average time delay of the model is analyzed and model-based simulation is conducted with Stochastic Petri Net Package (SPNP) 6.0 [9]. Finally, section 5 conclude our paper and variation trends of different parameters' effect on two troupes' average time delay were derived thereby. 


\section{Analysis of interaction process in typical manage systems for entertainment performance chains}

For typical manage systems for entertainment performance chains, there are a variety of interactive relationships troupes, theatres and audiences. Figure 1 gives the timing diagram of interaction process within related participants.

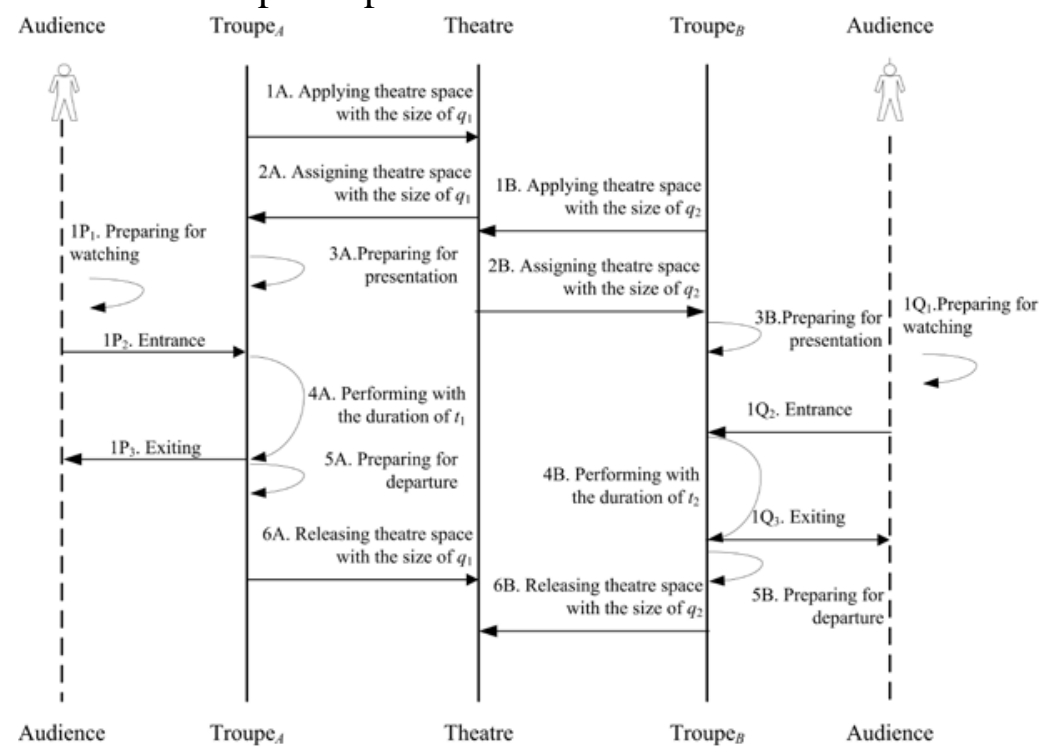

Figure 1: The timing diagram of interaction process among troupes, theatres and audiences.

As shown in Figure 1, there exist interactive behaviors among audiences, troupes and theatre. For one thing, audience and troupe collaborate during the period of presentation. For another, there doesn't exist constraint of time sequence in their respective steps. For the sake of demonstrating the mutual interactions among such participants, we symbolize the corresponding numbers for troupes in the form of "serial number" and "A" or "B". Similarly, the combination of "serial number", "P" or "Q" and subscript are used to sign different audiences. Still, from the viewpoint of participants, two audiences are almost identical when they interact with troupes. Moreover, TroupeA and TroupeB have the same process when they interact with theatre and audiences. Consequently, we simplify the introduction of timing diagram by considering TroupeA, audience and theatre. The detailed steps are shown as follows.

1A. TroupeA applies theatre space with the size of q1 from theatre.

2A. When the available space of theatre is larger than q1, theatre will assign space with the size of q1 to TroupeA.

3A. TroupeA prepares for the presentation and issues information about presentations and theatre via online and offline platforms.

1P1. Audience prepares for watching the presentation performed by TroupeA.

1P2. Audience enters the theatre which represents the constraints of time and space among audience, TroupeA and theatre.

4A. TroupeA performs the show with the duration of $\mathrm{t} 1$.

1P3. Audience exits after the presentation ends.

5A. TroupeA prepares for departure.

$6 \mathrm{~A}$. TroupeA releases theatre space with the size of q1.

It follows that the events of audiences' arrival and troupes' presentations have their respective sequences. Still the events have the characteristics of collaboration during the presentation for audiences and troupes. At the same time, TroupeA and TroupeB share theatre in a competitive and parallel manner.

\section{SPN model of two troupes' behaviors of sharing theatre with different resource needs}

Places and transitions are two basic types of elements of Petri net. The connection between place and place or between transition and transition is not allowed. A directed arc connecting a place 
(transition) to a transition (place) is called an input arc (output arc) of the transition. The number of tokens in a place is zero or positive integer. And a positive number can also be assigned to the multiplicity of corresponding input arc. The corresponding transition is enabled if each of its input places' tokens is no less than the multiplicity of an input arc.

Since the requirements of various troupes are different in acquiring theatre resources. For instance, a bigger troupe would occupy larger theatre space than that of a smaller one. Here, we denote the bigger troupe as TroupeA which occupies the size of q1. Moreover, the smaller troupe is signed as TroupeB which occupies the size of q2. For troupes, they all expect the average time delay of whole phase to be small and can fully utilize the space of theatre.

Further, we make the following assumptions.

i) The behaviors of audiences' arrivals follow the Poisson process.

ii)The actions of TroupeA and TroupeB's presentations are independent of each other, and follow the Poisson process.

We constructed the SPN model as shown in Figure 2. Let $\mathrm{q} 1=2 \mathrm{q}, \mathrm{q} 2=\mathrm{q}$. Here $\mathrm{q}$ represents the unit of standard theatre space.

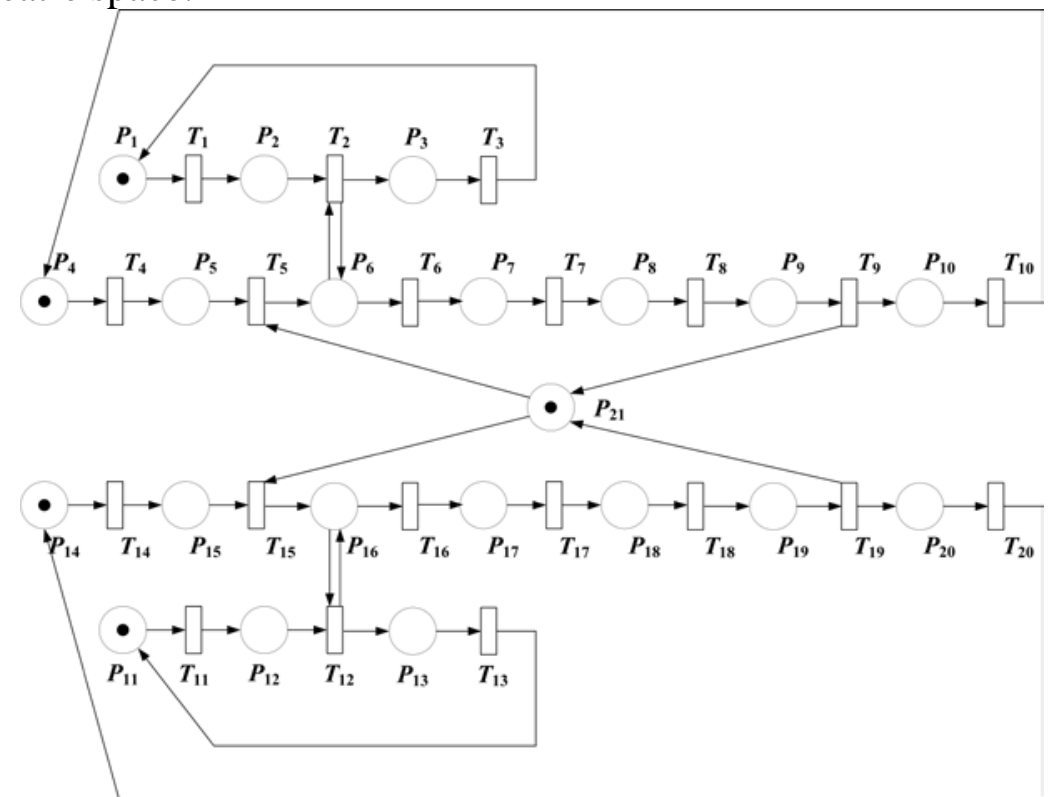

Figure 2: SPN model of two troupes' behaviors of sharing theatre with different resource needs.

As Fig. 2 indicates, four arcs reflect the phenomena of competition in using the shared theatre with different resource needs for TroupeA and TroupeB, which are the arc from T9 to P21, the one from P21 to T5, the one from T19 to P21 and the one from P21 to T15. Let the weights of first arc and second one be q1 which means TroupeA would occupy theatre space with the size of q1. Similarly, the weights of third arc and forth one be q2 which implies TroupeB would take up theatre space with the size of q2. The related sizes of theatre space won't be released until the troupes finish their respective presentations. In order to reflect the different demands of troupes, we assume that the space of TroupeA is larger than that of TroupeB, i.e. q1> q2. Obviously, the minimum value of theatre space is no smaller than q1. The idea theatre space is the sum of all troupes' requirements so that all of troupes can perform in parallel without any conflict. However, the chance of troupes being on show all the time is slim. Hence the design of theatre should take the efficient use of theatre space as one key factor.

Let “\#” symbolize the token number of a certain transition. The firing condition of T5 is formulated as follows: $\# P_{5}>0 \wedge \# P_{21} \geq q_{1}$. As such, the firing condition of T15 is formulated as follows: $\# P_{15}>0 \wedge \# P_{21} \geq q_{2}$.

The objects of the SPN model are listed in Table 1.

Table 1: List of Objects of SPN model of two Troupes’ Behaviors of Sharing Theatre with Different Resource Requirements

No Name Meaning $\quad$ Markin Rate




\begin{tabular}{|c|c|c|c|c|}
\hline & & & gs & \\
\hline 1 & $\begin{array}{l}P_{1} \\
P_{11}\end{array}$ & Audience prepares for watching & $N_{1}$ & \\
\hline 2 & $\begin{array}{l}T_{1} \\
T_{11}\end{array}$ & Audience watch the presentation & & $\lambda_{1}$ \\
\hline 3 & $\begin{array}{l}P_{2}, \\
P_{12}\end{array}$ & Audience’s arrival & 0 & \\
\hline 4 & $\begin{array}{l}T_{2} \\
T_{12}\end{array}$ & Watching the presentation & & $\lambda_{2}$ \\
\hline 5 & $\begin{array}{l}P_{3} \\
P_{13}\end{array}$ & Prepare for exiting & 0 & \\
\hline 6 & $\begin{array}{l}T_{3} \\
T_{13}\end{array}$ & Exiting from the theatre & & $\lambda_{3}$ \\
\hline 7 & $\begin{array}{l}P_{4} \\
P_{14}\end{array}$ & Troupe $_{A}$ or Troupe ${ }_{B}$ 's arrival & $N_{2}$ & \\
\hline 8 & $\begin{array}{l}T_{4} \\
T_{14}\end{array}$ & $\begin{array}{l}\text { Troupe }_{A} \text { or } \text { Troupe }_{B} \text { is ready to } \\
\text { present }\end{array}$ & & $\lambda_{4}$ \\
\hline 9 & $\begin{array}{l}P_{5} \\
P_{15}\end{array}$ & $\begin{array}{l}\text { applying theatre space with the } \\
\text { required size from theatre }\end{array}$ & 0 & \\
\hline 10 & $\begin{array}{l}T_{5} \\
T_{15}\end{array}$ & $\begin{array}{l}\text { Obtaining required size of theatre } \\
\text { space }\end{array}$ & & $\lambda_{5}$ \\
\hline 11 & $\begin{array}{l}P_{6} \\
P_{16}\end{array}$ & $\begin{array}{l}\text { Ready to present in the assigned } \\
\text { theatre }\end{array}$ & 0 & \\
\hline 12 & $\begin{array}{l}T_{6} \\
T_{16}\end{array}$ & $\begin{array}{l}\text { Synchronizing actions of groups } \\
\text { in Troupe }_{A} \text { or Troupe } \\
B\end{array}$ & & $\lambda_{6}$ \\
\hline 13 & $\begin{array}{l}P_{7} \\
P_{17}\end{array}$ & Ready to present formally & 0 & \\
\hline 14 & $\begin{array}{l}T_{7} \\
T_{17}\end{array}$ & Presenting the show & & $\lambda_{7}$ \\
\hline 15 & $\begin{array}{l}P_{8} \\
P_{18}\end{array}$ & Ready to finish the presentation & 0 & \\
\hline 16 & $\begin{array}{l}T_{8} \\
T_{18}\end{array}$ & finishing the presentation & & $\lambda_{8}$ \\
\hline 17 & $\begin{array}{l}P_{9} \\
P_{19}\end{array}$ & Ready to depart from theatre & 0 & \\
\hline 18 & $\begin{array}{l}T_{9} \\
T_{19}\end{array}$ & Departing from theatre & & $\lambda_{9}$ \\
\hline 19 & $\begin{array}{l}P_{10} \\
P_{20}\end{array}$ & $\begin{array}{l}\text { Ready to update the status of } \\
\text { Troupe }_{A} \text { or Troupe }_{B} \text { 's preparation }\end{array}$ & 0 & \\
\hline 20 & $\begin{array}{l}T_{10} \\
T_{20}\end{array}$ & $\begin{array}{l}\text { Storing related digital media to } \\
\text { theatre }\end{array}$ & & $\lambda_{10}$ \\
\hline 21 & $P_{21}$ & Shared theatre & $M$ & \\
\hline
\end{tabular}

\section{Performance evaluation}

We focus on the average time delay of TroupeA and TroupeB during the whole procedure of presentation. Further, through SPN reachability analysis and isomorphic continuous time Markov process equations with SPNP 6.0 software, we can calculate average time delay of the constructed SPN model by applying Little's theorem and principle of balance [10]. Additionally N2 represents the number of shows to be presented for two troupes. Then two experiments are designed to investigate different parameters' effects on average time delay.

Case 1: In the case of $\mathrm{N} 1=1, \mathrm{q} 1=2, \mathrm{q} 2=1, \lambda 1=\lambda 2=\lambda 3=0.2, \lambda 4=\lambda 5=0.3, \lambda 6=\lambda 7=\lambda 8=\lambda 9=\lambda 10=0.5$, 
the effect of number of presentations, size of theatre space on two troupes' average time delay is shown in Figure 3. Let N2=N. For the different parameters of $M=2,3$ and 4, six different series are depicted.

Due to the addition of presentations to be shown, it can be seen that in general for each troupe average time delay increases with the argument of $\mathrm{N}$. What's more, TroupeA competes with TroupeB in using shared theatre. Since the space of TroupeB is smaller than that of TroupeA, if there is not enough space for both Troupe $A$ and TroupeB simultaneously TroupeB is easier to gain the right of occupying the theatre. This would result in smaller queue delay for TroupeB. Subsequently, average time delay of Troupe $A$ is greater than that of TroupeB in most situations by comparing the curves of TroupeA and TroupeB. Besides upgrading the space of theatre would decrease or eliminate the chance of conflict for troupes in using shared theatre. Therefore it can also demonstrate that larger space of theatre result in obvious decline of average time delay. That is, average time delay decreases with M’s enhancement for both troupes.

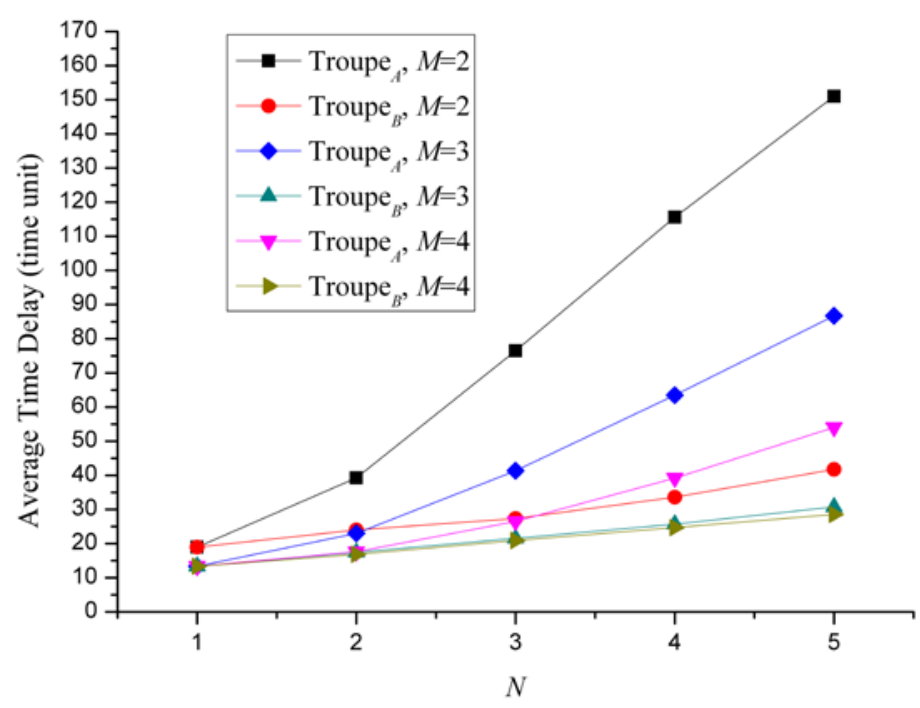

Figure 3: Effect of number of presentations, size of theatre space on two troupes’ average time delay.

Case 2: In the case of $N_{1}=1, N_{2}=3, \lambda_{1}=\lambda_{2}=\lambda_{3}=0.2, \lambda_{4}=\lambda_{5}=0.3, \lambda_{6}=\lambda_{7}=\lambda_{8}=\lambda_{9}=\lambda_{10}=0.5$, the effect of space requirements, size of theatre space on two troupes' average time delay is illustrated in Figure 4. For the different parameters of $M=10,13$ and 15, six different series are plotted. Let $q_{1}=2 q$ and $q_{2}=q$, where $q$ indicates the standard unit of theatre space.

Due to the addition of presentations to be shown, it can be seen that in general for each troupe average time delay increases with the argument of $\mathrm{N}$. Moveover with the raise of space requirements for each troupe average time delay augments gradually in the form of polygonal line. Further, for a certain q, average time delay of TroupeA is much bigger than that of TroupeB. The difference average time delay between and is largest when $M=10$ and $q=4$, which results from that under the circumstances theatre space is not enough for both TroupeA and TroupeB. Similarly, in the case of $M=13$, the average time delay of TroupeA becomes bigger rapidly when q varies from 4 to 5. In brief, the curves of TroupeA's average time delay contain two jump points where $q=4$ and $\mathrm{q}=5$.

In order to mine the mechanism of $\mathrm{M}$ and q's affections on average time delay for both troupes, we can find that there exists regular pattern of jump points. As for these points, average time delay increase drastically relative to former points. Since $q 1=2 \mathrm{q}$ and $\mathrm{q} 2=\mathrm{q}$, the parallel requirement of theatre space to offer TroupeA and TroupeB in sum is 3q. Once the theatre space is larger than $3 q$ which signifies two troupes can conduct their presentations independently. If $q \leq \mathrm{M}<2 \mathrm{q}$, only TroupeB can perform at theatre. Moreover, if $2 \mathrm{q} \leq \mathrm{M}<3 \mathrm{q}$, the queue delay will take a large proportion of average time delay. 
More specifically, bigger requirement of theater space leads to higher average time delay for troupes. For six series of curves in Figure 4, these jump points will appear once $q$ satisfy the inequality of " $2 \mathrm{q} \leq \mathrm{M}<3 \mathrm{q}$ ". We can infer that these jump points will arise when $q=\lfloor M / 3\rfloor+1$.

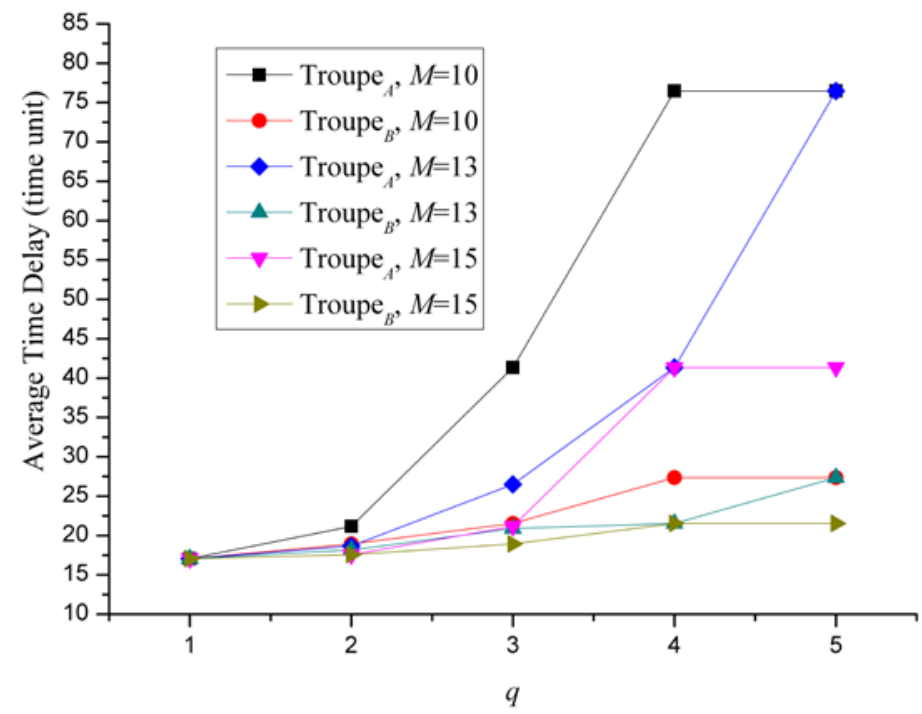

Figure 4: Effect of space requirements, size of theatre space on two troupes’ average time delay.

\section{Conclusion}

By focusing on the interaction process among troupes, theatres and audiences, this paper constructs a SPN model to evaluate two troupes' behaviors of sharing theatre with different resource needs. Two case studies are conducted to analyze different parameters' effects on average time delay for two troupes by comparison. Therefore, the trend that average time delay changes with number of presentations, size of theatre space are demonstrated. Also, affection of related parameters for both troupes is derived. Additionally, conclusion on some jump points, which reflects dramatic increase of two troupes' average time delay, is inferred by a formula thereby. Further works on analysis of collaborative objects in manage systems for entertainment performance chains can be carried on.

\section{Acknowledgements}

The research work was supported by the National Science and Technology Support Program (Foundation project: Research on the manage systems for entertainment performance chains and key technology of web collaboration services platform, Project number: 2012BAH02F04) and Engineering Project of Communication University of China (Project number: 3132014XNG1433).

\section{References}

[1] Seung-yun Kim. Modeling and analysis of a web-based collaborative enterprise using Petri nets. IEEE International Conference on Information Reuse and Integration, pp.422-428, 2008.

[2] Wang Yongbin, Liu Jing, Meng Yuejin, Meng Qinglong. Model of synchronous computer supported collaborative design in enterprise dynamic alliance. JOURNAL OF COMPUTERAIDED DESIGN\&COMPUTER GRAPHICS, 16(7), pp.984-991, 2004. (in chinese)

[3] Ferraro A.M., Rogers E.H. Petri nets in the evaluation of collaborative systems. IEEE International Conference on Systems, Man, and Cybernetics, Computational Cybernetics and Simulation., 4, pp. 3918-3923, 1997. 
[4] Kwangyeol Ryu, Yucesan E. A novel method of modeling collaborative processes in manufacturing. Proceedings of the Ninth International Conference on Computer Supported Cooperative Work in Design, 2, pp. 1082-1087, 2005.

[5]C. A. Petri: “Kommunkation mit automaten”, Bonn: Institut fur Lnstrum Entelle Mathematik, Schriften des IIM Nr. 3, 1962. Also English translation, “Communication with automata”[R]. Technical Report RADC-TR-65-377. Rome Air Dev. Center, New York, 1966.

[6] C. Xiong, T. Murata, and J. Tsai, Modeling and simulation of routing protocol for mobile ad hoc networks using Colored Petri Nets, Research and Practice in Information Technol., 12, pp. 145153, Australian Computer Society, 2002.

[7] J. Wise, J. Xia, C. K. Chang, and J. C. Huang, Performance analysis based on requirements traceability, Tech. Rep. 05-04, Dept. Computer Science, Iowa State Univ., 2005.

[8]M. A. Marson: Stochastic Petri Nets:an elementary Introduction. Lecture Notes in Computer Science, 424, New York: Springer-Verlag, 1989.

[9]C. Hirel, B. Tuffin, K. S. Trivedi: SPNP:Stochastic Petri Nets. version 6.0. Lecture Notes in Computer Science, 1786, pp. 354-357, 2000.

[10] C. Lin. Stochastic Petri Nets and System Performance Evaluation. Beijing: Tsinghua University Press, 2000. (in chinese) 